
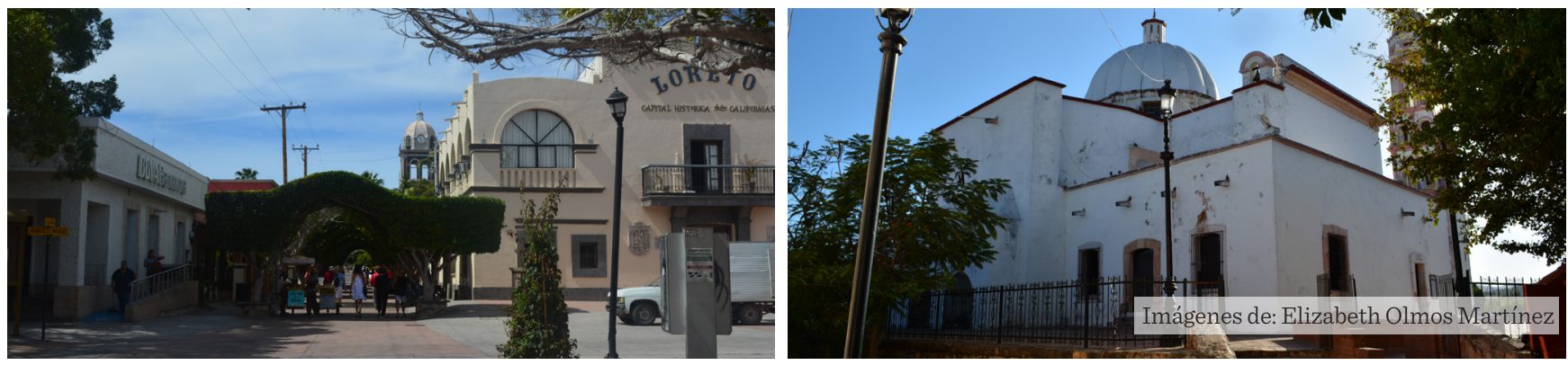

\title{
Atributos que influyen en la elección del visitante en cuatro pueblos mágicos del noroeste mexicano
}

\section{Attributes that influence the visitor's choice in four magical towns in the Mexican northwest}

\author{
Marco Antonio Almendarez-Hernández ${ }^{1}$, Reyna María Ibáñez-Pérez², Elizabeth Olmos-Martínez ${ }^{3 *}$
}

\section{RESUMEN}

El concepto pueblos mágicos fue ideado para incrementar el turismo. Se concentra en la difusión de actividades atractivas en pequeñas localidades con atributos histórico-culturales que representan la identidad nacional. El objetivo de esta investigación fue estimar la probabilidad de seleccionar un determinado pueblo mágico del noroeste mexicano a partir de factores tales como el perfil del usuario, su experiencia, percepción y satisfacción de los servicios y el gasto efectuado durante su estancia. La metodología utilizada se basa en el modelo econométrico Logit Multinomial y estadística descriptiva con variables de control para su análisis a partir de la aplicación de una encuesta en cuatro pueblos mágicos: Todos Santos y Loreto, de Baja California Sur, y Cosalá y El Rosario, de Sinaloa. Los resultados muestran que las probabilidades condicionadas de escoger uno de ellos son mayores para Todos Santos con $29 \%$, seguido de Cosalá con $28 \%$, Loreto con $27 \%$ y, finamente, El Rosario con $16 \%$. La variación de la elección está en función de las especificidades y características de los destinos. Los resultados del modelo permitirán la planeación de estrategias para mejorar la atención y la promoción turística en los cuatro pueblos mágicos estudiados, basándose en las preferencias de quienes eligen estos destinos, para atraer a más visitantes.

PALABRAS CLAVE: elección, atributos, visitante, pueblos mágicos, logit multinomial.

\section{ABSTRACT}

The magical towns concept was devised to increase tourism. It focuses on the dissemination of attractive activities in small towns with historical-cultural attributes that represent the national identity. The objective of this research was to estimate the probability of selecting a certain magical town in northwestern Mexico based on factors such as the user's profile, their experience, perception and satisfaction of the services and the expenditure made during their stay. The methodology used is based on the Multinomial Logit econometric model and descriptive statistics with control variables was used for its analysis. The survey was administered in four magical towns: Todos Santos and Loreto, from Baja California Sur, and Cosalá and El Rosario, from Sinaloa. Results show that the probabilities of choosing one of them are higher for Todos Santos with $29 \%$, followed by Cosalá with $28 \%$, Loreto with $27 \%$ and, finally, El Rosario with $16 \%$. The variation of the choice is a function of the specificities and characteristics of the destinations. The results of the model will allow the planning and strategies to improve tourist service and promotion in the four magical towns studied, based on the preferences of those who choose these destinations, to attract more visitors.

KEYWORDS: choice, attributes, visitor, magical towns, multinomial logit.

*Correspondencia: eolmosm.udo@gmail.com/ Fecha de recepción: 15 de febrero de 2021/Fecha de aceptación: 25 de junio de 2021/Fecha de publicación: $2^{2}$ de julio de 2021 .

${ }^{1}$ Centro de Investigaciones Biológicas del Noroeste SC (CIBNOR), Subcoordinación de Proyectos Productivos Socialmente Responsables y Extensionismo Social, La Paz, Baja California Sur, México. ${ }^{2}$ Universidad Autónoma de Baja California Sur, Departamento Académico de Economía, La Paz Baja California Sur, México. ${ }^{3}$ Universidad Autónoma de Occidente, Departamento Académico de Ciencias Económicoadministrativas, Unidad Mazatlán, Av. Del Mar núm. 1200, col. Flamingos, Mazatlán, Sinaloa, México, C. P. 82149. 


\section{INTRODUCCIÓN}

: Según datos del Diario Oficial de la Federación (DOF, 2014), el programa de Pueblos Mágicos (PM) fue creado en 2001 con la finalidad de impulsar a México como un destino a nivel mundial mediante la promoción turística basada en la riqueza del patrimonio material e inmaterial del país, fomentando la coordinación interinstitucional y el lazo con el estado y municipios, a manera de diversificar los servicios turísticos y fomentar la inversión pública y privada, para generar derrama económica y empleo.

Por otra parte, la Organización Mundial del Turismo señala que este se caracteriza por ser multidimensional, configurable y heterogéneo ante cualquier espacio y circunstancia. Además, guarda relación con los servicios y bienes que demandan directamente los turistas y con las unidades económicas que lo generan y suministran (Pérez-Ramírez y Antolín-Espinosa, 2016). Por ello, es importante conocer el perfil (variables socioeconómicas y demográficas de los visitantes, tales como gustos, preferencias, expectativas y necesidades), ya que es una herramienta para mejorar la gestión de las empresas al igual que del destino turístico (Pat y Calderón, 2012; Huang y Wang, 2018; Lin y Kuo, 2018). En ese sentido, de acuerdo con Pons y col. (2007); Cohen y col. (2014); Possebon y col. (2019), de las decisiones que tiene que tomar el turista y/o visitante potencial, la elección del destino es la más importante, por lo que es pertinente identificar y señalar los elementos que intervienen en ella. Los mismos autores mencionan que el más influyente es la imagen del destino, ya que se convierte en un atractivo. Cuando el potencial visitante perciba una imagen relacionada con la satisfacción de sus necesidades y deseos de ocio, ese destino tendrá mayores posibilidades de ser seleccionado.

Castaño y col. (2006), así como Stylidis y col. (2017) refieren que, desde el punto de vista psicológico, el destino turístico es una construcción mental basada en un proceso de evaluación de la información que el visitante potencial adquiere de diferentes fuentes y que el re- sultado de ese proceso es lo que se conoce como imagen-destino, un factor determinante en la elección de lugares de ocio y recreación. Autores como Folgado y col. (2011) y Castro y col. (2019) indican que la imagen es la clave para atraer a los turistas, lo clasifican como elemento crítico en la elección del destino; asimismo, aseguran que dicha imagen es independiente de si es o no representativa de lo que ofrece.

Galarza y Soria (2018) argumentan que la toma de decisión sobre el destino se maneja en un entorno altamente competitivo, y obliga al posible turista a una continua actualización en el manejo de la tecnología. El nivel de información virtual, a través de la motivación emocional, influye en la percepción de los consumidores cuando buscan un destino en función de sus gustos y prioridades. Esto significa que el marketing es otro de los elementos fundamentales en la decisión, tal como lo destacan Mediano (2002) y Martos (2015) en el estudio de la incidencia del consumidor turístico a partir de un análisis de influencia del modelo estímulo-respuesta en su comportamiento.

También Lazzari y col. (2009), así como Tan y Wu (2016) consideran que, debido a la variedad de atributos que se involucran en la elección del sitio, no todas las opciones despiertan el mismo interés, ya que existen factores subjetivos que influyen directamente; no obstante, aquellos destinos que satisfacen los criterios de búsqueda iniciales del individuo forman el conjunto de elementos con las cuales se ha familiarizado, los recuerda y fueron evaluados positivamente; es entonces cuando el consumidor toma la decisión final.

En la literatura existen diversos estudios que abordan los determinantes de elección del destino turístico, tal es el caso de Albaladejo y Díaz (2003) y De-la-Hoz y Muñoz (2016), quienes argumentan factores del perfil del turista (viajar con amigos, tipo de alojamiento, edad, ingresos familiares, nivel de estudios, referencias del lugar, entre otros); además, De-la-Mata (2010) y Kastenholz y col. (2018) mencionan que un factor de decisión es si el viaje lo hacen dentro 
de la región donde viven o fuera de ella, por lo que el factor origen-destino tiene una gran relevancia. Por su lado, Ercolano y col. (2018) afirman que los sitios con renombre o fama mundial por su riqueza arqueológica y patrimonial son mayormente elegidos, independientemente de que existan otros sitios de valor arqueológico similar pero de menor renombre. Saladié y col. (2014) indican que el costo de los boletos de avión interviene notablemente en la elección; asimismo, De-Conto y col. (2011) observan que las condiciones de saneamiento básico del destino turístico son un factor de elección del visitante; Domínguez y de-Sevilha (2017) y Santana y Gosling (2017) destacan que un sitio puede ser más exitoso y estar asociado a una mayor predilección por el visitante en función de la prevalencia de una imagen única como destino; también, Da-Cruz y De-Camargo (2008) señalan que las estrategias de promoción en la web influyen en la decisión. Galarza y Soria (2018) manifiestan que valores familiares, creencias religiosas, seguridad y confort son también factores importantes; finalmente, Choque (2017) refiere que las personas de la generación millennial consideran diferentes elementos de decisión que el resto de la población para la elección de un destino turístico, tal como vivir nuevas experiencias y conocer gente de otro lugar.

De acuerdo con el Sistema nacional de información estadística del sector turismo de México (DATATUR, 2020a; 2020b; 2020c; 2020d, 2020e), para el año 2020 el número de PM asciende a 131, de los cuales 9 pertenecen a la región noroeste del país, zona conformada por Baja California, Baja California Sur, Sonora y Sinaloa, estados que se caracterizan por tener una localización estratégica, vastos litorales, buen nivel de vida y enorme afluencia de visitantes; en conjunto recibieron a 13.9 millones al cierre de 2018, que representa $34 \%$ del total nacional durante ese periodo.

El objetivo de esta investigación fue estimar la probabilidad de que se escoja un determinado pueblo mágico del noroeste mexicano de los cuatro que fueron tomados como caso de estudio Cosalá y El Rosario, de Sinaloa, y Loreto y Todos Santos, de Baja California Sur, a partir de las características del visitante, tales como su perfil, la experiencia, percepción y satisfacción de los servicios y el gasto efectuado durante su estancia.

\section{MATERIALES Y MÉTODOS}

La estimación de la probabilidad de que el visitante seleccione un determinado PM de la región noroeste de México se realizó a partir de ciertas variables explicativas, como el motivo del viaje, las experiencias y satisfacciones acerca del trayecto y de los servicios y actividades que ofrece el lugar. La información se obtuvo mediante una encuesta compuesta por dos secciones: a) perfil del visitante (género, procedencia, rangos de edad, con quién viaja y medio de transporte) y b) evaluación de atributos de satisfacción (cultural, hospedaje, transporte, actividades, satisfacción, destino, caminata, diversidad y gasto). El instrumento fue validado con una prueba piloto efectuada en marzo de 2019. La duración máxima fue de 10 min, cara a cara, se dirigió solamente a mayores de edad con una estadía mínima de $3 \mathrm{~h}$ en el lugar de estudio, considerando que en ese lapso el visitante ya tuvo la oportunidad de observar, contratar o adquirir algún servicio turístico que le permitió percibir los atributos estudiados. Las encuestas se aplicaron durante los meses de marzo y abril de 2019 a visitantes de los PM de Loreto y Todos Santos (en Baja California Sur), y de Cosalá y El Rosario (en Sinaloa). El tipo de muestreo fue aleatorio estratificado con afijación proporcional y se calcula mediante la fórmula siguiente (CochranGemmel, 1989):

$$
n=\frac{\sum_{i=1}^{I} N_{i} P_{i} Q_{i}}{N E+\frac{1}{N} \sum_{i=1}^{I} N_{i} P_{i} Q_{i}}
$$

Donde $\eta$ es el tamaño de la muestra, $N i$ es la población de cada estrato, $P_{i}$ es la proporción, $Q i$ es el complemento de la proporción, $\mathrm{N}$ es el tamaño de la población, $\mathrm{E}$ es el error de estimación. El tamaño de cada estrato se calcula por medio de la siguiente fórmula (CochranGemmel, 1989): 


$$
n_{i}=n\left(\frac{N_{i}}{\sum_{i=1}^{I} N_{i}}\right)=n\left(\frac{N_{i}}{N}\right)=n\left(W_{i}\right)
$$

Donde Wi es la fracción de asignación. El tipo de muestreo es aleatorio, porque todos los elementos que comprenden el universo tienen la misma probabilidad de ser escogidos en el tamaño de la muestra calculado, de acuerdo a la proporción que fue estimada para cada estrato (PM), además de que se escoge al azar el número de elementos que está determinado para cada uno. En este sentido, se debe elegir por lo menos un elemento de cada estrato, para que tenga representatividad. La determinación del tamaño se basó a partir de un total de 367946 visitantes registrados anualmente en los cuatro destinos, con base en la disposición de datos de fuentes oficiales e investigación de campo, dividiéndose de esta manera: 1) Todos Santos con 126074 (Ibáñez y Cruz, 2016); 2) Loreto con 107218 (Gobierno del Estado de Baja California Sur-INEGI, 2017); 3) Cosalá con 90 189, de acuerdo con la Secretaría de Turismo (SECTUR, 2017); 4) El Rosario con 59 7775, según información del Consejo de Desarrollo de Sinaloa (CODESIN, 2018). El total de encuestas fue de 415, las cuales se repartieron en los cuatro sitios: Loreto con 116; Todos Santos con 136; Cosalá con 98 y El Rosario con 65. Los participantes completaron todas las preguntas del cuestionario, por lo que el número de encuestas válidas fueron las que se calcularon.

Acorde con McFadden (1974a; 1974b) y Train (1998; 2009), la Teoría de la Maximización de la Utilidad Aleatoria es el marco teórico usado para analizar las decisiones de elección de los individuos por destinos o modos de viaje. El análisis empírico se basa en los trabajos de Albaladejo y Díaz (2003); De-la-Mata (2010); Ercolano y col. (2018); Nurlaela (2018); Yoo y col. (2018), que han usado como especificación econométrica el modelo Logit Multinomial.

La función de utilidad tiene dos componentes: a) Uno determinístico, que incluye la utilidad observada de la alternativa que está en fun- ción de las variables de control ya mencionadas; b) el segundo es un término estocástico, el cual recoge los elementos no observables de la función de utilidad del individuo. Si se establece la suposición de que un visitante $i$ escoge un conjunto que incluye $j$ alternativas de PM $(j=1,2,3,4)$ y que se puede extender hasta el j-ésimo PM, 1 representa a Todos Santos, 2 a Loreto, 3 a El Rosario, 4 a Cosalá. Bajo este planteamiento el visitante $i$ escoge la alternativa que maximiza su utilidad. Si expresa $Y_{i j}$ como una variable respuesta de elección discreta que toma el valor de 1 y el visitante elija el PM de Todos Santos solamente, y toma el valor de 0 si es otro sitio. A modo de ejemplo, se puede mencionar que un visitante que maximiza su utilidad escogerá su primera opción, es decir, el PM de Todos Santos, solamente si se cumple la desigualdad siguiente:

$$
\begin{aligned}
& Y_{i 1}=1 \text { Si } U_{i 1}>U_{i j}, j=2,3,4 \\
& Y_{i 1}=0 \text { en caso contrario }
\end{aligned}
$$

La probabilidad de que el visitante $i$ seleccione la primera alternativa del destino $d$ (el PM de Todos Santos) se puede expresar de esta forma:

$$
P_{i 1}=\operatorname{Pr}\left(U_{i 1}>U_{i 2}, U_{i 1}>U_{i 3}, U_{i 1}>U_{i 4}, \ldots, U_{i 1}>U_{i a}\right)_{(4)}
$$

Donde Uij es la función de utilidad del visitante $i$ para la alternativa $j$, y, siguiendo a Maddala (1983); Powers y Xie (2000); Dow y Endersby (2004), se expresa como:

$$
U_{i j}=\beta^{\prime} X_{i j}+\delta^{\prime} Z_{i}+\varepsilon_{i j}
$$

$X i j$ representa un vector de atributos de las alternativas, $Z i$ es un vector de variables de las características de los individuos, $\beta$ se refiere a un conjunto de parámetros que están relacionados con los atributos, $\delta$ describe un conjunto de parámetros asociados con las características de los individuos, eij es el término estocástico que captura los efectos no observados pero que influyen en la función de utilidad. 
La probabilidad de que el visitante $i$ escoja el destino $d$ en el conjunto de alternativas se expresa como:

$$
\begin{aligned}
P(d)= & \operatorname{Pr}\left\{\varepsilon_{i j}-\varepsilon_{i d}<\left(\beta^{\prime} X_{i j}+\delta_{j}^{\prime} Z_{i}\right)-\right. \\
& \left.\left(\beta^{\prime} X_{i d}+\delta_{d}^{\prime} Z_{i}\right), j \neq d\right\}
\end{aligned}
$$

De acuerdo con McFadden (1974a; 1974b) y Train (1998), el término estocástico está independiente e idénticamente distribuido y sigue una distribución logística (Gumbel I). Si $\varepsilon_{i j}^{*}=\varepsilon_{i j}-\varepsilon_{i d} . \mathrm{y} V_{i j}^{*}=\left(\beta^{\prime} X_{i j}+\delta_{j}^{\prime} Z_{i}\right)-\left(\beta^{\prime} X_{i d}+\delta_{d}^{\prime} Z_{i}\right)$ con $\forall j \neq d$, entonces el modelo que expresa la alternativa de referencia es el PM de Todos Santos es:

$$
\begin{aligned}
& P_{d}(i)=\frac{1}{1+\sum_{j=1}^{J} \exp \left(\beta_{j}^{\prime} z_{j}\right)} \quad \text { para todo } j \in J, j \neq d \\
& P_{d}(i)=\frac{\exp \left(\beta_{j}^{\prime} z_{j}\right)}{1+\sum_{j=1}^{J} \exp \left(\beta_{j}^{\prime} z_{j}\right)} \quad \text { para todo } j \in J, j \neq d
\end{aligned}
$$

Los coeficientes del modelo Logit Multinomial son estimados con máxima verosimilitud, sin embargo, el modelo Logit Multinomial presenta limitaciones como la violación del supuesto de Independencia de Alternativas Irrelevantes (IIA, por sus siglas en inglés: Independence from Irrelevant Alternatives). Esto es debido a que su uso complica el modelo de elección discreta, es decir, las características de la alternativa que ha elegido el individuo impactan en las probabilidades relativas del resto de las alternativas que se escojan. Cuando se viola esta propiedad, el modelo Logit Multinomial genera estimaciones de los coeficientes que son sesgados e inconsistentes. Para verificar la validez del supuesto IIA se calcula la prueba de Hausman (Hausman y McFadden, 1984), que consiste en contrastar las diferencias entre un modelo restringido y otro no restringido. El estadístico está distribuido como $\chi^{2}$. En este sentido, si se mantiene el supuesto IIA, la hipótesis nula de la prueba de Hausman (Hausman y McFadden, 1984) indica que la estimación del modelo Logit Multinomial no debe provocar cambios sistemáticos en los coeficientes si una alternativa es incluida o excluida.

\section{RESULTADOS Y DISCUSIÓN}

El perfil del visitante contextualiza las características de los sujetos de estudio que visitaron los cuatro PM (Tabla 1).

La estadística descriptiva, junto con la descripción de cada una de las variables usadas en el análisis de regresión se muestran en la Tabla 2. El modelo fue estimado en el Paquete estadístico Stata versión 15.1 (StataCorp, 2017). Dado que el tratamiento econométrico es un modelo Logit Multinomial y esta especificación plantea el supuesto IIA, se recurrió a la prueba de Hausman (Hausman y McFadden, 1984), arrojando un resultado con valor $\mathrm{P}$ de 0.591 relacionado a la prueba $\chi^{2}$. Este valor indica que no se puede rechazar la hipótesis nula de IIA y sugiere que los coeficientes estimados son insesgados y consistentes.

En la regresión que engloba los cuatro PM (Tabla 3), Todos Santos es la variable respuesta categórica usada como referencia omitida, para el análisis de regresión, ya que fue el destino que registró el mayor número de visitantes en el año en que se aplicó la encuesta. Los hallazgos del modelo son respaldados en la literatura que aborda los determinantes de la elección del destino, en el sentido de que los signos de los coeficientes son acordes con lo que señala la teoría económica y muestra la evidencia empírica (Albaladejo y Díaz, 2003; Lyons y col., 2009; Ahn y col., 2013; Pagliara y col., 2015; Almeida y Garrod, 2018; Ercolano y col., 2018; Masiero y Qiu, 2018; Nurlaela, 2018; Yoo y col., 2018). La prueba de Wald es estadísticamente significativa al $1 \%$ e indica que el modelo global es mejor que aquel que contiene solo la intersección. El pseudo- $\mathrm{R}^{2}$ se encuentra dentro del rango de los obtenidos por la evidencia empírica de estudios de elección del destino (Dela-Mata, 2010; Ercolano y col., 2018; Li y col., 2019) y señala que aproximadamente el $18.1 \%$ de la variación de la alternativa PM es explicada por el modelo estimado. Las probabilidades condicionadas de elegir cada uno de los cuatro pueblos mágicos son las siguientes: 1) Todos Santos con $29 \%$; 2) Loreto con $27 \%$; 3) El Rosario con $16 \%$ y; 4) y Cosalá con $28 \%$ (Tabla 4). 
Tabla 1. Perfil del visitante de los cuatro pueblos mágicos.

Table 1. Visitor profile of the four magical towns.

\begin{tabular}{|c|c|c|c|c|}
\hline \multirow{2}{*}{ Perfil } & Loreto & Todos Santos & El Rosario & Cosalá \\
\hline & \multicolumn{4}{|c|}{ Opciones del perfil / Porcentaje de visitantes } \\
\hline \multirow{3}{*}{ Género } & Femenino: $49 \%$ & Femenino: $47 \%$ & Femenino: $47 \%$ & Femenino: $56 \%$ \\
\hline & Masculino: $50 \%$ & Masculino: $53 \%$ & Masculino: $53 \%$ & Masculino: $44 \%$ \\
\hline & Otro: $1 \%$ & Otro: $0 \%$ & Otro: $0 \%$ & Otro: $0 \%$ \\
\hline \multirow{4}{*}{ Procedencia } & México: $29 \%$ & México: $72 \%$ & México: $93 \%$ & México: $92 \%$ \\
\hline & USA: $46 \%$ & USA: $17 \%$ & USA: $3 \%$ & USA: $8 \%$ \\
\hline & Canadá: $16 \%$ & Canadá: $4 \%$ & Canadá: $2 \%$ & Canadá: $0 \%$ \\
\hline & Otro: $9 \%$ & Otro: $7 \%$ & Otro: $2 \%$ & Otro: $0 \%$ \\
\hline \multirow{7}{*}{$\begin{array}{l}\text { Rangos de edad } \\
\text { (años) }\end{array}$} & 18 a $25: 9 \%$ & 18 a $25: 19 \%$ & 18 a $25: 26 \%$ & 18 a $25: 33 \%$ \\
\hline & 26 a $33: 10 \%$ & 26 a $33: 9 \%$ & 26 a $33: 16 \%$ & 26 a $33: 15 \%$ \\
\hline & 34 a $41: 4 \%$ & 34 a $41: 19 \%$ & 34 a $41: 22 \%$ & 34 a $41: 22 \%$ \\
\hline & 42 a $48: 5 \%$ & 42 a $48: 15 \%$ & 42 a $48: 7 \%$ & 42 a $48: 14 \%$ \\
\hline & 49 a $55: 15 \%$ & 49 a $55: 17 \%$ & 49 a $55: 15 \%$ & 49 a $55: 12 \%$ \\
\hline & 56 a $63: 13 \%$ & 56 a $63: 10 \%$ & 56 a $63: 11 \%$ & 56 a $63: 1 \%$ \\
\hline & 64 y más: $44 \%$ & 64 y más: $11 \%$ & 64 y más: $3 \%$ & 64 y más: $3 \%$ \\
\hline \multirow{5}{*}{ Con quién viaja } & Cónyuge: $38 \%$ & Cónyuge: $19 \%$ & Cónyuge: $6 \%$ & Cónyuge: $14 \%$ \\
\hline & Familia: $30 \%$ & Familia: $39 \%$ & Familia: $55 \%$ & Familia: $57 \%$ \\
\hline & $\begin{array}{l}\text { Compañeros de } \\
\text { trabajo: } 3 \%\end{array}$ & $\begin{array}{l}\text { Compañeros de } \\
\text { trabajo: } 11 \%\end{array}$ & $\begin{array}{l}\text { Compañeros de } \\
\text { trabajo: } 8 \%\end{array}$ & $\begin{array}{l}\text { Compañeros de } \\
\text { trabajo: } 9 \%\end{array}$ \\
\hline & Amigos: $19 \%$ & Amigos: $20 \%$ & Amigos: $27 \%$ & Amigos: $19 \%$ \\
\hline & Solo: $10 \%$ & Solo: $11 \%$ & Solo: $4 \%$ & Solo: $1 \%$ \\
\hline \multirow{5}{*}{$\begin{array}{l}\text { Medio de } \\
\text { transporte }\end{array}$} & Auto propio: $35 \%$ & Auto propio: $69 \%$ & Auto propio: $64 \%$ & Auto propio: $69 \%$ \\
\hline & Auto rentado: $25 \%$ & Auto rentado: $19 \%$ & Autobús: $20 \%$ & Autobús: $20 \%$ \\
\hline & Avión: $22 \%$ & Autobús: $6 \%$ & Auto rentado: $7 \%$ & Auto rentado: $5 \%$ \\
\hline & Autobús: $5 \%$ & Avión: $2 \%$ & Ninguno: $5 \%$ & Ninguno: $5 \%$ \\
\hline & Otro: 13 & Otro: $4 \%$ & Otros: $4 \%$ & Otros: $1 \%$ \\
\hline
\end{tabular}

Tabla 2. Estadística descriptiva de las variables de control.

Table 2. Descriptive statistics of the control variables.

\begin{tabular}{|l|c|c|}
\hline \multicolumn{1}{|c|}{ Variable } & Descripción & Media \\
\hline Cultural & $\begin{array}{r}\text { Variable dicotómica que toma el valor de } 1 \text { si la razón principal que motivó el } \\
\text { viaje del individuo fue cultural y 0 en caso contrario }\end{array}$ & 0.063 \\
\hline Hospedaje & $\begin{array}{c}\text { Variable dicotómica que toma el valor de } 1 \text { si los servicios de hospedaje fueron } \\
\text { calificados por el individuo como excelentes y 0 en caso contrario }\end{array}$ & 0.337 \\
\hline Transporte & $\begin{array}{c}\text { Variable dicotómica que toma el valor de } 1 \text { si el individuo usó transporte propio y } \\
\text { calificó la carretera y señalamiento como excelentes y 0 en caso contrario }\end{array}$ & 0.190 \\
\hline Actividades & $\begin{array}{c}\text { Variable dicotómica que toma el valor de } 1 \text { si el individuo no practicó actividades } \\
\text { recreativas y de esparcimiento y 0 en caso contrario }\end{array}$ & 0.096 \\
\hline Satisfacción & $\begin{array}{c}\text { Variable dicotómica que toma el valor de } 1 \text { si el nivel de satisfacción del turista al } \\
\text { retirarse del sitio es calificado como excelente y 0 en caso contrario }\end{array}$ & 0.398 \\
\hline Destino & $\begin{array}{c}\text { Variable dicotómica que toma el valor de } 1 \text { si el individuo se enteró del sitio por } \\
\text { medio de la familia y 0 en caso contrario }\end{array}$ & 0.289 \\
\hline
\end{tabular}

Continúa... 


\begin{tabular}{|l|c|c|}
\hline Caminata & $\begin{array}{r}\text { Variable dicotómica que toma el valor de } 1 \text { si el individuo practicó la caminata } \\
\text { por el sitio y } 0 \text { en caso contrario }\end{array}$ & 0.569 \\
\hline Diversidad & $\begin{array}{c}\text { Variable dicotómica que toma el valor de } 1 \text { si el individuo calificó como mala la } \\
\text { experiencia de la diversidad de las actividades y 0 en caso contrario }\end{array}$ & 0.080 \\
\hline Gasto 1 & $\begin{array}{r}\text { Variable dicotómica que toma el valor de } 1 \text { si el gasto promedio por persona al día } \\
\text { es menor o igual a } \$ 500 \text { pesos y } 0 \text { en caso contrario }\end{array}$ & 0.313 \\
\hline Gasto 2 & $\begin{array}{r}\text { Variable dicotómica que toma el valor de } 1 \text { si el gasto promedio por persona al día } \\
\text { se encuentra entre } \$ 501 \text { y } \$ 1000 \text { pesos y } 0 \text { en caso contrario }\end{array}$ & 0.340 \\
\hline Gasto 3 & $\begin{array}{r}\text { Variable dicotómica que toma el valor de } 1 \text { si el gasto promedio por persona al día } \\
\text { se encuentra entre } \$ 1001 \text { y } \$ 1500 \text { y } 0 \text { en caso contrario }\end{array}$ & 0.137 \\
\hline Gasto 4 & $\begin{array}{r}\text { Variable dicotómica que toma el valor de } 1 \text { si el gasto promedio por persona al día } \\
\text { es mayor a } \$ 1500 \text { pesos y } 0 \text { en caso contrario }\end{array}$ & 0.210 \\
\hline
\end{tabular}

Tabla 3. Resultados del modelo Logit Multinomial.

Table 3. Results of the Multinomial Logit Model.

\begin{tabular}{|c|c|c|c|c|c|c|}
\hline \multirow{2}{*}{ Variables } & \multicolumn{2}{|c|}{ Loreto } & \multicolumn{2}{|c|}{ El Rosario } & \multicolumn{2}{|c|}{ Cosalá } \\
\hline & Coeficiente & z-estadístico & Coeficiente & z-estadístico & Coeficiente & z-estadístico \\
\hline Constante & $-1.345^{* * *}$ & -3.11 & 0.405 & 1.06 & $-1.171^{* * *}$ & -2.85 \\
\hline Cultural & $-3.014^{* * * *}$ & -2.97 & $-1.563^{* *}$ & -2.27 & -0.833 & -1.46 \\
\hline Hospedaje & $1.155^{* * *}$ & 3.47 & -0.224 & -0.55 & $0.830^{* *}$ & 2.37 \\
\hline Transporte & $-0.611^{*}$ & -1.64 & -0.533 & -1.18 & $-1.224^{* * *}$ & -3.04 \\
\hline Actividades & -0.795 & -1.43 & $1.197^{* * *}$ & 2.65 & $-1.410^{*}$ & -1.88 \\
\hline Satisfacción & $0.990^{* * *}$ & 3.15 & -0.522 & -1.34 & $0.666^{* *}$ & 2.00 \\
\hline Destino & 0.442 & 1.24 & 0.069 & 0.2 & $0.785^{* *}$ & 2.31 \\
\hline Caminata & 0.106 & 0.34 & 0.482 & 1.55 & 0.503 & 1.63 \\
\hline Diversidad & $1.314^{* *}$ & 1.97 & $1.782^{* * *}$ & 2.89 & -.594 & -0.65 \\
\hline Gasto 1 & Referencia & & & & & \\
\hline Gasto 2 & 0.391 & 0.99 & $-1.043^{* * *}$ & -2.84 & 0.407 & 1.07 \\
\hline Gasto 3 & 0.713 & 1.41 & $-1.232^{* * *}$ & -2.56 & 0.323 & 0.64 \\
\hline Gasto 4 & $1.596^{* * *}$ & 3.51 & $-2.525^{* * *}$ & -2.77 & $1.6101^{* * *}$ & 3.44 \\
\hline $\begin{array}{l}\text { Log } \\
\text { likelihood }\end{array}$ & -471.282 & & & & & \\
\hline $\begin{array}{l}\text { Log } \\
\text { likelihood } \\
\text { restringido }\end{array}$ & -575.222 & & & & & \\
\hline $\begin{array}{l}\text { Wald } \\
\operatorname{chi}^{2}(33)\end{array}$ & 125.66 & & & & & \\
\hline Pseudo $R^{2}$ & 0.181 & & & & & \\
\hline
\end{tabular}

*Significancia al 10\%, ${ }^{* *}$ Significancia al 5\%, ${ }^{* * *}$ Significancia al 1\%. 
Tabla 4. Efectos marginales del modelo Logit Multinomial.

Table 4. Marginal Effects of the Multinomial Logit Model.

\begin{tabular}{|c|c|c|c|c|c|c|c|c|}
\hline \multirow[b]{2}{*}{ Variables } & \multicolumn{2}{|c|}{ Todos Santos } & \multicolumn{2}{|c|}{ Loreto } & \multicolumn{2}{|c|}{ El Rosario } & \multicolumn{2}{|c|}{ Cosalá } \\
\hline & 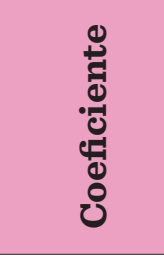 & 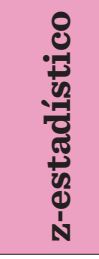 & 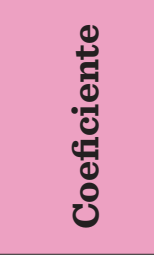 & 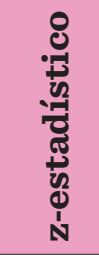 & 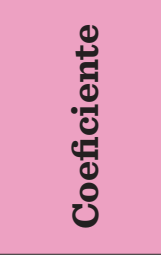 & 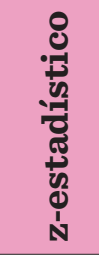 & 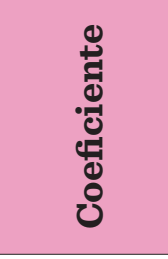 & 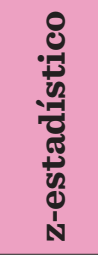 \\
\hline Cultural & $0.350^{* * *}$ & 3.17 & $-0.265^{* * *}$ & -5.91 & $-0.086^{*}$ & -1.65 & 0.000 & 0.00 \\
\hline Hospedaje & $-0.144^{* * *}$ & -2.77 & $0.176^{* * *}$ & 3.2 & $-0.111^{* * * *}$ & -2.61 & 0.079 & 1.37 \\
\hline Transporte & $0.179^{* * *}$ & 2.4 & -0.022 & -0.38 & -0.001 & -0.03 & $-0.156^{* * *}$ & -3.14 \\
\hline Actividades & 0.017 & 0.2 & $-0.145^{* *}$ & -2.43 & $0.351^{* * *}$ & 3.85 & $-0.224^{* * *}$ & -3.9 \\
\hline Satisfacción & $-0.107 * *$ & -2.05 & $0.168^{* * *}$ & 3.27 & $-0.136^{* * *}$ & -3.59 & 0.075 & 1.38 \\
\hline Destino & $-0.099^{*}$ & -1.85 & 0.020 & 0.36 & -0.046 & -1.25 & $0.125^{* * *}$ & 2.17 \\
\hline Caminata & -0.072 & -1.36 & -0.037 & -0.75 & 0.038 & 1.01 & 0.070 & 1.45 \\
\hline Diversidad & $-0.170^{* *}$ & -2.35 & 0.159 & 1.54 & $0.237^{* *}$ & 2.51 & $-0.227^{* * *}$ & -4.11 \\
\hline Gasto 2 & -0.022 & -0.36 & 0.086 & 1.22 & $-0.157^{* * *}$ & -4.1 & 0.093 & 1.34 \\
\hline Gasto 3 & -0.053 & -0.66 & $0.166^{*}$ & 1.7 & $-0.149^{* * *}$ & -4.38 & 0.036 & 0.41 \\
\hline Gasto 4 & $-0.175^{* * *}$ & -3.28 & $0.222^{* * *}$ & 2.73 & $-0.280^{* * *}$ & -9.15 & $0.233^{* * * *}$ & 2.78 \\
\hline $\begin{array}{l}\text { Probabilidades } \\
\text { condicionadas }\end{array}$ & \multicolumn{2}{|c|}{$29 \%$} & \multicolumn{2}{|c|}{$27 \%$} & \multicolumn{2}{|c|}{$16 \%$} & \multicolumn{2}{|c|}{$28 \%$} \\
\hline
\end{tabular}

*Significancia al $10 \%,{ }^{* *}$ Significancia al $5 \%,{ }^{* * *}$ Significancia al $1 \%$.

Nota: se omite la variable Gasto 1 por el problema de la trampa de la variable dicotómica.

El análisis de la probabilidad de que los individuos elijan alguno de los cuatro PM se efectúa a través de los efectos marginales (Tabla 4), los cuales, para un modelo Logit Multinomial, son interpretados como variaciones en las probabilidades predichas por el efecto de los cambios en las variables de control analizadas (Cameron y Trivedi, 2005). La discusión de los resultados se centra solamente en aquellos coeficientes que resultaron estadísticamente significativos a los niveles usuales, que son $\mathrm{P}$ valores de 0.01, 0.05 y 0.10 .

En el caso de Todos Santos, el motivo del viaje con fines de disfrutar eventos culturales (variable cultural) impacta positivamente en esta opción y aumenta la probabilidad en $35 \%$ de elegir este pueblo. Para Loreto y El Rosario el efecto marginal cultural es negativo, con disminuciones en las probabilidades de $27 \%$ y $9 \%$, respectivamente. Estos hallazgos se deben a que Todos Santos, por su tradición, es preferido por la cultura que representa, mientras que en los otros dos pueblos el principal interés es por motivos de descanso. Las investigaciones de Ercolano y col. (2018) y Yoo y col. (2018) señalan que el efecto tiende a variar dependiendo de lo que el turista busque encontrar en el sitio para disfrutar del mismo. Lyons y col. (2009) encuentran en su estudio que los viajeros irlandeses que prefieren áreas con patrimonios culturales inciden negativamente en la elección de un país como destino de vacaciones. Almeida y Garrod (2018) demuestran que quienes se inclinan por descubrir nuevas culturas y formas de vida afectan 
positivamente la opción de un destino costero con vasta experiencia en la recepción de turistas.

Los individuos que calificaron los servicios de hospedaje como excelentes afectan negativamente en las elecciones de Todos Santos y El Rosario, mientras que en Loreto el efecto es positivo. La disminución de las probabilidades es del orden de $14 \%$ y $11 \%$ para los 2 primeros pueblos y para Loreto un aumento de $18 \%$. La razón es que Loreto cuenta con servicios de hospedaje de más categoría que Todos Santos y Cosalá, por tratarse de un polo turístico de hegemonía que comprende una infraestructura hotelera considerable. Las personas que tienden a gastar más en alojamiento prefieren este lugar y, por ende, la puntuación que le otorgan a los servicios de hospedaje es más alta (Albaladejo y Díaz, 2003). Los estudios de Ahn y col. (2013) y Masiero y Qiu (2018) mencionan que los turistas seleccionan el sitio por la disponibilidad y la calidad de los servicios.

Los visitantes que usaron transporte propio y consideraron la carretera y señalamientos como excelentes (variable transporte) tuvieron un efecto negativo en Cosalá, con una disminución en la probabilidad de $16 \%$; y una influencia positiva en Todos Santos, con un aumento de $18 \%$. La diferencia en el signo de las relaciones se asocia a que la carretera que transita a Todos Santos es una autopista y con señalamientos muy bien definidos, a diferencia de Cosalá. El trabajo de Li y col. (2019) indica que si el lugar escénico en el sitio se muestra en la señalización, este predictor influye positivamente en la visita. Los viajeros que no se interesan en las actividades recreativas y de esparcimiento (variable actividades) son menos propensos a elegir Loreto y Cosalá, con disminuciones en las probabilidades de $15 \%$ y de $22 \%$, respectivamente, mientras que se inclinan más por El Rosario, con aumento de $35 \%$. La disparidad en los signos de los efectos se atribuye a que Loreto tiene una gran oferta de actividades recreativas acuáticas como la pesca deportiva, el buceo, el kayaking y veleros; en Cosalá los atractivos son el eco- turismo y la aventura. En lo que respecta a El Rosario, las propuestas que existen en este sitio para que los viajeros lo visiten son de tipo cultural y las festividades, sin embargo los turistas manifestaron que este motivo de viaje se relaciona negativamente con la elección de este PM. El trabajo de Yoo y col. (2018) menciona que la influencia de esta variable de control en signo puede cambiar si el turista prefiere o no las actividades recreativas y de esparcimiento. Por su parte, Masiero y Qiu (2018) argumentan que los visitantes que calificaron las atracciones recreativas al aire libre como excelentes inciden positivamente en la selección del destino. Almeida y Garrod (2018) muestran evidencia de que quienes disfrutan de la aventura y el placer tienden a elegir un destino costero con vasta experiencia en la recepción de turistas.

Los individuos que otorgaron un nivel de excelencia a la experiencia que tuvieron en su visita (variable satisfacción) son menos proclives a elegir Todos Santos y El Rosario, disminuyendo sus probabilidades en $11 \%$ y $14 \%$, respectivamente. El efecto marginal es positivo sobre la elección de Loreto, aumentándolas en $17 \%$. La disimilitud en las influencias de los signos se debe a que Loreto les ofrece una gama de servicios turísticos más amplia que el resto de los destinos. Las investigaciones de Ahn y col. (2013) y Masiero y Qiu (2018) señalan que el atractivo de la atmósfera que incluya restaurantes, hoteles, monumentos, lugares históricos, facilidades de transportación, escenarios naturales y entretenimiento influye sustancialmente en la elección del lugar.

Los visitantes que recibieron información sobre el sitio por medio de la familia (variable destino) mostraron un impacto negativo en la elección de Todos Santos, disminuyendo la probabilidad en 10 \%. En lo que respecta a Cosalá, su asociación con esta variable es positiva, incrementándola en $13 \%$. Estos resultados se explican en que quiénes eligen Todos Santos, su principal medio de referencia son los amigos. Los visitantes que calificaron como mala la experiencia de la variedad de actividades (va- 
riable diversidad) son menos propensos a elegir Todos Santos y Cosalá, con disminuciones en sus probabilidades de $17 \%$ y $23 \%$, respectivamente. En El Rosario la relación con esta variable explicativa es positiva, aumentándola en $24 \%$. El razonamiento para el contraste en los signos se asocia a que en Todos Santos y Cosalá es limitada la pluralidad de las actividades, mientras que en El Rosario, aunque le faltan más atractivos turísticos, los visitantes lo consideran como uno de sus sitios de gran preferencia por su cultura y festividades. De acuerdo con Ercolano y col. (2018), dependiendo del listado de actividades a desarrollar el visitante dentro del sitio, el individuo elegirá un destino.

La variable Gasto 1 (menor o igual a $\$ 500$ pesos) se usa como referencia (Tabla 4) para la estimación de la regresión y se interpretan los resultados con base en ésta porque si es incluida en la estimación se presenta el problema de la trampa de la variable dicotómica. Esta categoría de gasto es contrastada con los estratos Gasto 2 (entre $\$ 501$ pesos y $\$ 1000$ pesos), Gasto 3 (entre $\$ 1001$ pesos y $\$ 1500$ pesos) y Gasto 4 (mayor a $\$ 1500$ pesos). Los viajeros que se concentran en estos tres últimos rangos de desembolsos tienen menores probabilidades de elegir el PM El Rosario, con un registro del $16 \%, 15 \%$ y $28 \%$, respectivamente. En cuanto a Todos Santos, solamente los visitantes del nivel de Gasto 4 disminuyen en $18 \%$ su probabilidad de seleccionarlo. En lo que respecta a Loreto, los de los Gastos 3 y 4 aumentan sus probabilidades de elegir este sitio con $17 \%$ y $22 \%$, respectivamente; mientras que en Cosalá, solo aquellos del Gasto 4 las aumentan en $23 \%$. Los resultados de Todos Santos y El Rosario son acordes con los trabajos de Eymann y Ronning (1997); Pagliara y col. (2015); Nurlaela (2018); Masiero y Qiu (2018). Sin embargo, en los casos de Loreto y Cosalá, los hallazgos se atribuyen a que una gran proporción de los individuos que eligen estos sitios gastan por arriba de los $\$ 1500$ pesos, implicando una estadía de aproximadamente una semana, donde el desembolso contempla el hotel. En el estudio de Albaladejo y
Díaz (2003) este factor tiene un efecto positivo y considera como gasto el correspondiente a alojamiento. En Todos Santos y El Rosario su visita por lo general es de paso, tendiendo a efectuar un gasto más bajo, de acuerdo a los resultados que arrojó la encuesta en el componente de gasto y estadía.

Los resultados obtenidos proporcionan información útil para que los tomadores de decisiones y prestadores de servicios turísticos puedan implementar acciones que lleven a mejorar la competitividad de sus negocios y la imagen del destino en general, elementos importantes que pueden influir en el desarrollo y la calidad de vida de la población local. Lo anterior puede coadyuvar a un mayor involucramiento de los residentes, como ha sido documentado en diversos estudios, como Pérez y col. (2011); Velázquez y Clausen (2012); Carrillo (2015); Madrid (2019). En consecuencia, esto forjará una percepción más positiva tanto del visitante como del residente con relación a los beneficios de la actividad turística (Mendoza y González, 2014). Por lo contrario, de acuerdo con Mendoza (2012); Carrillo (2013); Duarte (2015); Enciso (2015), si dichas estrategias no integran la participación activa de la población local, se puede correr el riesgo de exacerbar la apreciación de impactos negativos.

Las limitaciones del presente estudio se relacionan con que no es factible segmentar la muestra entre extranjeros y nacionales, dado que los primeros representan el $29 \%$. En este sentido, dado que en El Rosario y Cosalá los extranjeros constituyen el $11 \%$ y $8 \%$, respectivamente, no sería posible estimar el modelo debido a que habría variabilidad insuficiente en los valores de las regresoras. Otro inconveniente es que la aplicación de la encuesta en la temporada de primavera podría afectar la estabilidad de los resultados, ya que el perfil de los visitantes podría ser diferente a los de otros períodos.

\section{CONCLUSIONES}

Al estimar la perspectiva de que el visitante escoja un determinado PM de la región noro- 
este de México a partir de atributos como el perfil del visitante, la experiencia de los servicios, la percepción de los mismos, su satisfacción y el gasto efectuado durante su estancia arrojó que las probabilidades condicionadas de decidirse por cada uno de los cuatro PM son mayores en Todos Santos, seguido de Cosalá, Loreto y finamente, El Rosario. Al analizar los cuatro PM se encontró que las probabilidades de elección del visitante como destino turístico varían en función de las especificidades del sitio, por ejemplo, en Todos Santos el motivo del viaje con fines de disfrutar eventos culturales impacta positivamente e incrementa la posibilidad de seleccionarlo, mientras que para sitios como Loreto y El Rosario se observa mayor predilección por realizar actividades de descanso. Estos datos permiten conocer la per- cepción de los tipos de atributos que ofrece cada PM, para que cada uno de ellos mejore la promoción y la atención de los mismos para seguir satisfaciendo las preferencias de los turistas como su lugar de destino, así como proponer o mejorar otros atractivos, para ofrecer actividades dirigidas a visitantes con otro perfil y preferencias. Las investigaciones futuras pueden dirigirse a manejar cuestionarios que incluyan una mayor cantidad de ítems del perfil del turista y más PM, con un tamaño de muestra que permita segmentar entre visitantes nacionales y extranjeros, donde exista un monitoreo de aplicación de la encuesta interanual. Esta investigación podría ser complementada con la incorporación de metodologías más cualitativas que se enfoquen a las preferencias de la elección de los PM.

\section{REFERENCIAS}

Ahn, T., Ekinci, Y., and Li, G. (2013). Self-congruence, functional congruence, and destination choice. Journal of Business Research. 66(6): 719-723.

Albaladejo I. y Díaz, M. (2003). Un modelo de elección discreta en la determinación del perfil del turista rural: Una aplicación a Murcia. Cuadernos de Turismo.11: 7-19.

Almeida, A. and Garrod, B. (2018). A CATREG model of destination choice for a mature Island destination. Journal of Destination Marketing \& Management. 8: 32-40.

Cameron, C. and Trivedi, P. (2005). Microeconometrics. Methods and applications. United Kingdom: Cambridge University Press. 1034 Pp.

Carrillo, A. (2013). Pueblos Mágicos y la gestión del turismo en México. Caso Cuetzalan, Puebla (2002-2011). Tesis de Licenciatura en Ciencias Políticas y Administración Pública. UNAM. 100 Pp.

Carrillo, A. (2015). Pueblos mágicos, de la magia al desarrollo local. México: Buen Gobierno. 19: 106-119.

Castaño, J. M., Moreno, A. y Crego, A. (2006). Factores psicosociales y formación de imágenes en el turismo urbano: un estudio de caso sobre Madrid. Pasos. 4(3): 287299.

Castro, J. C., Palacios, J. M. y Plazarte, L. (2019). Imagen del destino desde la perspectiva del turista. Turismo y Sociedad. 26: 45-66.

Choque, M. (2017). Determinantes en la decisión del millennial para elegir el destino: Bolivia (Tesis de licenciatura). Universidad Mayor de San Andrés, La Paz Bolivia.
[En línea]. Disponible en: https://repositorio.umsa.bo/bits tream/handle/123456789/12817/TG-3983.pdf?sequence=1. Fecha de consulta: 22 de febrero de 2021.

Cochran-Gemmel, W. (1989). Sampling Techniques. Nueva York: Willey and Sons. 428 Pp.

CODESIN, Consejo de Desarrollo de Sinaloa (2018). Reporte de llegada de turistas y ocupación hotelera en Sinaloa, de enero a diciembre de 2017. Unidad de estadística y análisis. [En línea]. Disponible en: http://sinaloaennume ros.com/wp-content/uploads/2018/01/Reporte-07-del2018-de-turismo-a-ene-dic.-2017.pdf. Fecha de consulta: 22 de febrero de 2021.

Cohen, S. A., Prayag, G., and Moital, M. (2014). Consumer behaviour in tourism: concepts, influences and opportunities. Current Issues in Tourism. 17(10): 872-909.

Da-Cruz, G. y De-Camargo, P. (2008). Estrategias de promoción en la web. Análisis de destinos turísticos internacionales. Estudios y Perspectivas en Turismo. 17(2): 156-169.

DATATUR, Sistema Nacional de Información Estadística del Sector Turismo (2020a). Baja California. Llegada de Turistas totales a la Entidad. [En línea]. Disponible en: https://www.datatur.sectur.gob.mx/ITxEF/ITxEF_BCN.aspx. Fecha de consulta: 21 de febrero de 2021.

DATATUR, Sistema Nacional de Información Estadística del Sector Turismo (2020b). Baja California Sur. Llegada de Turistas totales a la Entidad. [En línea]. Disponible en: https://www.datatur.sectur.gob.mx/ITxEF/ITxEF_ 
BCS.aspx. Fecha de consulta: 21 de febrero de 2021.

DATATUR, Sistema Nacional de Información Estadística del Sector Turismo (2020c). Sonora. Llegada de Turistas totales a la Entidad. [En línea]. Disponible en: https:// www.datatur.sectur.gob.mx/ITxEF/ITxEF_SON.aspx. Fecha de consulta: 21 de febrero de 2021.

DATATUR, Sistema Nacional de Información Estadística del Sector Turismo (2020d). Sinaloa. Llegada de Turistas totales a la Entidad. [En línea]. Disponible en: https:// www.datatur.sectur.gob.mx/ITxEF/ITxEF_SIN.aspx. Fecha de consulta: 21 de febrero de 2021.

DATATUR, Sistema Nacional de Información Estadística del Sector Turismo (2020e). Anuncia la Secretaría de Turismo once nuevos Pueblos Mágicos. [En línea]. Disponible en: https://www.gob.mx/sectur/prensa/anuncia-la-secretaria-de-turismo-once-nuevos-pueblos-magicos. Fecha de consulta: 22 de febrero de 2021.

De-Conto, S. M., Costa-Alvez, T. J., Zaro, M., Pistorello, J., Pereira, G. S. y Pessin, R. (2011). Las condiciones de saneamiento básico como factor decisivo en la elección del destino turístico. Estudios y Perspectivas en Turismo. 20(1): 213-228.

De-la-Hoz, A. y Muñoz, F. (2016). Análisis de los determinantes de la predisposición a visitar un destino de turismo de bienestar: tipologías del turista potencial. Tourism \& Management Studies.12(2): 84-95.

De-la-Mata, T. (2010). La elección de destino según las características de las comunidades autónomas de residencia. Cuadernos de Economía. 33(91): 127-158.

DOF, Diario Oficial de la Federación (2014). Acuerdo por el que se establecen los Lineamientos generales para la incorporación y permanencia al Programa Pueblos Mágicos. [En línea]. Disponible en: https://www.dof.gob.mx/no ta_detalle.php? codigo $=5361690 \&$ fecha $=26 / 09 / 2014$. Fecha de consulta: 13 de enero de 2021.

Domínguez, L. y de-Sevilha, M. (2017). Unique Image of a Destination: exclusive attributes of the destination of Ilhéus, BA, Brazil. Revista Turismo em Análise - RTA. 28(1): 71-90.

Dow, J. K. and Endersby, J. W. (2004). Multinomial probit and multinomial logit: a comparison of choice models for voting research. Electoral Studies. 23(1):107-122.

Duarte, E. (2015). La participación del sector privado en la definición de la política urbana: el caso de la gestión del Programa Pueblos Mágicos en Cuitzeo, México. Revista de Estudios Urbanos y Ciencias Sociales. 5(2): 127-142.

Enciso, J. (2015). Real del Monte, Hidalgo iUna experiencia de éxito? En L. López, C. Valverde y M. Figueroa (Eds.), Pueblos Mágicos. Una visión interdisciplinaria (pp. 135-158). México, D.F.: UAM Xochimilco-UNAM.

Ercolano, S., Gaeta, G., and Parenti, B. (2018). Pompeii dilemma: A motivation-based analysis of tourists' preference for "superstar" archaeological attractors or less renowned archaeological sites in the Vesuvius area. International Journal of Tourism Research. 20(3): 345-354.

Eymann, A. and Ronning, G. (1997). Microeconometric models of tourists' destination choice. Regional Science and Urban Economics. 27(6): 735-761.

Folgado, J. A., Oliveira, P. A., and Hernández, J. M. (2011). Imagen del destino y marca turística: sinergias e implicaciones. Tourism and Management Studies. 1: 904-901.

Galarza, A. E. y Soria, S. M. (2018). Determinantes en la elección del destino turístico dentro de la Provincia de Tungurahua. Universidad Técnica de Ambato, Ambato, Ecuador. [En línea]. Disponible en: https: //repositorio.uta. edu.ec/bitstream/123456789/27663/1/682\%20MKT.pdf. Fecha de consulta: 26 de enero de 2021.

Gobierno del Estado de Baja California Sur-INEGI (2017). Anuario estadístico y geográfico de Baja California Sur 2017. [En línea]. Disponible en: http://internet.conte nidos.inegi.org.mx/contenidos/Productos/prod_serv/ contenidos/espanol/bvinegi/productos/nueva_estruc/ anuarios_2017/702825094881.pdf. Fecha de consulta: 22 de febrero de 2021.

Hausman, J. A. and McFadden, D. (1984). Specification Tests for the Multinomial Logit Model. Econometrica. 52: $1377-1398$

Huang, W. and P. Wang (2018). All that's best of dark and bright: Day and night perceptions of Hong Kong cityscape. Tourism Management. 66:274-286.

Ibáñez, R. y Cruz, P. (2016). Nivel de satisfacción del turista y necesidades de capacitación en un pueblo mágico. En E. Madrid (Ed.), Pueblos mágicos aciertos y retos (pp. 231-255). México: Editorial Limusa.

Kastenholz, E., Eusébio, C., and Carneiro, M. (2018). Segmenting the rural tourist market by sustainable travel behaviour: Insights from village visitors in Portugal. Journal of Destination Marketing \& Management. 10:132-142.

Lazzari, L. L., Mouliá, P. I., Fernandez, M. J., Chiodi, J. A., Chelmicki, F., Chiodi, M. y Eriz, M. (2009). Preferencias del segmento joven en la elección de un destino turístico. Cuadernos del CIMBAGE. (11): 59-84.

Li, Y., Yang, L., Shenc, H., and Wu, Z. (2019). Modeling intra-destination travel behavior of tourists through spatiotemporal analysis. Journal of Destination Marketing \& Management. 11: 260-269.

Lin, C. H. and Kuo, B. (2018). The moderating effects of travel arrangement types on tourists' formation of Tai- 
wan's unique image. Tourism Management. 66: 233-243.

Lyons, S., Mayor, K., and Tol, R. (2009). Holiday destinations: Understanding the travel choices of Irish tourists. Tourism Management.30(5): 683-692.

Maddala, G. S. (1983). Limited-Dependent and Qualitative Variables in Econometrics. New York: Cambridge University Press. $401 \mathrm{Pp}$.

Madrid, F. (2019). Derivaciones epistémicas de una política pública: el caso de los Pueblos Mágicos 2001-2015. El Periplo Sustentable. (36): 184-229.

Martos, L. (2015). Marketing en el sector turístico. España, Editorial Síntesis. 250 Pp.

Masiero, L. and Qiu, R. (2018). Modeling reference experience in destination choice. Annals of Tourism Research.72: 58-74.

McFadden, D. (1974a). Conditional logit analysis of qualitative choice behavior. In P. Zarembka (Ed.), Frontiers in Econometrics (pp. 105-142). New York: Academic Press.

McFadden, D. (1974b). The measurement of urban travel demand. Journal of Public Economics. 3: 303-328.

Mediano, L. (2002). Incidencia del nuevo consumidor turístico en la estrategia de marketing. Revista de Dirección y Administración de Empresas. (10): 99-117.

Mendoza, L. (2012). Análisis para la mejora en la aplicación del Programa Pueblos Mágicos en el municipio de Dolores Hidalgo, Guanajuato. Tesis de Licenciatura en Ciencias Políticas y Administración Pública. UNAM. 95 Pp.

Mendoza, M. y González, J. (2014). Impactos socioculturales del turismo en el Centro Integralmente Planeado Loreto, Baja California Sur, México. Percepción de los residentes locales. Teoría y Praxis. 16: 117-146.

Nurlaela, S. (2018). An application of Multinomial Logit Model (MNL) on tourist destination choices. IOP Conference Series: Earth and Environmental Science. 22: 1-8.

Pagliara, F., La-Pietra, A., Gomez, J., and Vassallo, J. (2015). High Speed Rail and the tourism market: Evidence from the Madrid case study. Transport Policy. 37: 187-194.

Pat, L. A. y Calderón, G. (2012). Caracterización del perfil turístico en un destino emergente, caso de estudio de Ciudad del Carmen, Campeche. México. Gestión Turística. (18): 47-70.

Pérez, C., Zizumbo, L., Romero, T., Cruz, G. y Madrigal, D. (2011). El turismo como intervención e implicaciones para las comunidades rurales. Gestión Turística. 16: 229-264.

Pérez-Ramírez, C. A. y Antolín-Espinosa, D. I. (2016). Programa pueblos mágicos y desarrollo local: actores, dimensiones y perspectivas en El Oro, México. Estudios Sociales. 25(47): 219-243.
Pons, R. C., Morales, L. y Díaz, Y. (2007). La imagen del destino y el comportamiento de compra del turista. Teoría y Praxis. 3: 89-102.

Possebon, J., Cervi, C. y Knebel, D. (2019). Factores que influyen en la decisión de compra de viajes turísticos un estudio en el Noroeste de Rio Grande Do Sul (Brasil). Estudios y Perspectivas en Turismo. 28: 903-922.

Powers, D. A. and Xie, Y. (2000). Statistical Methods for Categorical Data Analysis, New York: Academic Press. $295 \mathrm{Pp}$.

Saladié, O., Antón, S., Cortés-Jiménez, I., Fernandez, A. y Young, R. (2014). La influencia de las rutas de vuelos de bajo coste en la elección del destino turístico. Cuadernos de Turismo. (34): 287-312.

Santana, L. and Gosling, M. (2017). Unique image of a destination: Exclusive attributes of The case of Kavala. Journal of Travel Research.55(5): 659-674.

SECTUR, Secretaría de Turismo (2017). Principales indicadores turísticos, Cuarto trimestre 2015-2016, Sinaloa. [En línea]. Disponible en: http://www.transparenciasinaloa. gob.mx/images/stories/ST/programas\%20y\%20servicios/estadisticas/2016/TURISMO-INDICADORES_4to. Trim.2015-2016.pdf. Fecha de consulta: 22 de febrero de 2021.

StataCorp (2017). Stata: Release 15.1 (Versión 15.1) [Software de computo]. Texas, Estados Unidos de América: Stata Corp LLC.

Stylidis, D., Shani, A., and Belhassen, Y. (2017). Testing an integrated destination image model across residents and tourists. Tourism Management. 58: 184-195.

Tan, W. K. and Wu, C. E. (2016). An investigation of the relationships among destination familiarity, destination image and future visit intention. Journal of Destination Marketing \& Management. 5(3):214-226.

Train, K. (1998). Recreation demand models with taste differences over people. Land Economics. 74(2): 230-239.

Train, K. (2009). Discrete Choice Methods with Simulation. New York: Cambridge University Press. 388 Pp.

Velázquez, M. y Clausen, H. (2012). Tepoztlán, una economía de la experiencia íntima. Latin American Research Review. 47(3): 134-154.

Yoo, C. K., Yoon, D., and Park, E. (2018). Tourist motivation: an integral approach to destination choices. Tourism Review. 73(2): 169-185. 